\title{
Acidentes com motocicletas na Bahia: análise de uma década de internações hospitalares
}

\author{
Motorcycle accidents in Bahia: analysis of a decade of hospital admissions \\ Epidemia de accidentes con motocicletas en Bahía: análisis de una década de \\ internaciones hospitalarias
}

Thiago Batista Faleiro ${ }^{1 *}$, Augusto Morgan Alves Ladeia ${ }^{1}$, Antônio Manuel Pinto Júnior ${ }^{1}$, Renata da Silva Schulz², Davi Araújo Veiga Rosário ${ }^{1}$, Bruno Adelmo Ferreira Mendes Franco $^{2}$, Alexandre Vasconcelos de Meirelles ${ }^{1}$, Gildásio Daltro², Ademario Galvão Spínola ${ }^{2}$

\section{RESUMO}

Objetivo: Analisar as internações por acidentes com motocicletas no sistema público de saúde da Bahia no período entre 2008 e 2017. Métodos: Estudo transversal, descritivo e retrospectivo das internações por acidentes com motocicletas na rede pública de saúde da Bahia. Os dados foram obtidos do Sistema de Informações Hospitalares do SUS (SIH/SUS) do Ministério da Saúde. Resultados: Entre os anos de 2008 e 2017 foram realizadas 45714 internações decorrentes do grupo de causas do CID-10 V20-V29 (motociclista traumatizado em acidente de transporte). O tempo médio de internação foi de 31,12 dias e o custo total foi de aproximadamente 207 milhões e 798 mil reais. A faixa etária mais acometida foi a de 20 a 39 anos de idade representando $58,53 \%$ do total e o sexo masculino representou $85,12 \%$ das vítimas. A taxa de mortalidade foi em média de $9,54 \%$ refletindo a gravidade dos acidentes. Conclusão: $O$ estudo mostra a gravidade dos acidentes de motocicletas atendidos no Sistema Único de Saúde na Bahia comprometendo principalmente homens jovens, em idade produtiva e com alto custo e taxa de mortalidade. Esses dados reforçam a necessidade de medidas institucionais para redução do número de acidentes e novos estudos são importantes para determinar as sequelas que eventualmente acometem os sobreviventes.

Palavras chave: Acidentes de trânsito, Motocicletas, Traumatismo, Ortopedia.

\begin{abstract}
Objective: To analyze hospitalizations for accidents with motorcycles in the public health system of Bahia between 2008 and 2017. Methods: A cross-sectional, descriptive and retrospective study of hospitalizations for motorcycle accidents in Bahia's public health network. Results: Between 2008 and 2017, 45714 hospitalizations were performed, resulting from the group of causes of ICD-10 V20-V29 (a motorcyclist traumatized in transport accident). The average length of hospital stay was 31.12 days and the total cost was approximately 207 million and 798 thousand reais. The most affected age group was 20 to 39 years of age, representing $58.53 \%$ of the total and the male sex represented $85.12 \%$ of the victims. The mortality rate averaged $9.54 \%$ reflecting the severity of the accidents. Conclusion: The study shows the severity of accidents of motorcycles attended in the Unified Health System in Bahia involving mainly young men of productive age and with a high cost and mortality rate. These data reinforce the need for institutional measures
\end{abstract}

\footnotetext{
${ }^{1}$ Hospital Universitário Professor Edgard Santos, Salvador, Bahia, Brasil. *E-mail: thiagofaleiro@yahoo.com.br

${ }^{2}$ Universidade Federal da Bahia, Salvador, Bahia, Brasil.
}

SUBMETIDO EM: 12/2018

ACEITO EM: 1/2019

PUBLICADO EM: 6/2019

REAS/EJCH | Vol.Sup.24 | e1135 | DOI: https://doi.org/10.25248/reas.e443.2019 Página 1 de 7 
to reduce the number of accidents and new studies are important in determining the sequelae that eventually affect survivors.

Key words: Traffic accidents, Motorcycles, Traumatism, Orthopedics.

\section{RESUMEN}

Objetivo: Analizar las internaciones por accidentes con motocicletas en el sistema público de salud de Bahía en el período entre 2008 y 2017. Métodos: Estudio transversal, descriptivo y retrospectivo de las internaciones por accidentes con motocicletas en la red pública de salud de Bahía. Los datos fueron obtenidos del Sistema de Información Hospitalaria del SUS (SIH / SUS) del Ministerio de Salud. Resultados: Entre los años 2008 y 2017 se realizaron 45714 internaciones derivadas del grupo de causas del CID-10 V20-V29 (motociclista traumatizado en accidente de transporte). El tiempo promedio de internación fue de 31,12 días y el costo total fue de aproximadamente 207 millones y 798 mil reales. La franja etaria más acometida fue la de 20 a 39 años de edad representando el $58,53 \%$ del total y el sexo masculino representó el $85,12 \%$ de las víctimas. La tasa de mortalidad fue en promedio de 9,54\% reflejando la gravedad de los accidentes. Conclusión: El estudio muestra la gravedad de los accidentes de motocicletas atendidos en el Sistema Único de Salud en Bahía comprometiendo principalmente a hombres jóvenes, en edad productiva y con alto costo y tasa de mortalidad. Estos datos refuerzan la necesidad de medidas institucionales para reducir el número de accidentes y nuevos estudios son importantes para determinar las secuelas que eventualmente acomete a los sobrevivientes.

Palabras clave: Accidentes de tráfico, Motocicletas, Traumatismo, Ortopedia.

\section{INTRODUÇÃO}

Segundo o art. $1^{\circ}, \S 1^{\circ}$ do Código de Trânsito Brasileiro: "Considera-se como trânsito a utilização das vias por pessoas, veículos, animais, isolados ou em grupos, conduzidos ou não, para fins de circulação, parada, estacionamento e operação de carga ou descarga" (Código de Trânsito Brasileiro).

Considerado um dos países com o trânsito mais violento do mundo, nos últimos anos o Brasil tenta conter o alto número de acidentes. Desde a implantação do novo Código de Trânsito Brasileiro (CTB) em 1998, a taxa de mortalidade mantém-se estável (em torno de 20 mortes por 100 mil habitantes), superior às taxas do Japão, Suécia e Canadá (de cinco a oito mortes/100 mil habitantes) (BACCHIERI, BARROS, 2011).

Segundo A Organização Mundial de Saúde cerca 1,35 milhão de pessoas morrem a cada ano em decorrência de acidentes no trânsito e entre 20 e 50 milhões de pessoas sofrem lesões não fatais, muitas delas resultando em incapacidade. Os acidentes de trânsito custam à maioria dos países $3 \%$ de seu produto interno bruto (PIB) e são a principal causa de morte entre crianças e jovens de 5 a 29 anos. Além disso, 66.6\% de todas as mortes no trânsito ocorre entre usuários vulneráveis das vias: pedestres, ciclistas e motociclistas e $93 \%$ das mortes no trânsito ocorrem em países de baixa e média renda, embora estes concentrem aproximadamente 60\% dos veículos do mundo. (WORLD HEALTH ORGANIZATION, 2018).

As motocicletas invadiram o espaço urbano como ferramentas eficientes de transporte e trabalho diante do trânsito congestionado das grandes cidades. A ineficiência do transporte coletivo, o mercado de entregas, a possibilidade de renda para jovens sem qualificação profissional e a facilidade de aquisição de uma motocicleta contribuíram para crescimento em cinco vezes, em relação ao aumento da frota de automóveis. Existem mais de 14 milhões de motocicletas em circulação, o que corresponde a $25 \%$ da frota nacional. A motocicleta tornou-se o meio de transporte individual mais popular do Brasil (MARTINEZ, 2006).

Segundo dados da Associação Brasileira de Medicina de Tráfego (ABRAMET) as mortes causadas por acidentes com motos têm tido aumento superior às provocadas por outras doenças como as cardiovasculares e por neoplasias malignas. 
Segundo a Associação, no ano de 2006, ocorreram no Brasil pelo menos 6.655 mortes decorrentes de acidentes envolvendo motos, $20 \%$ do total de mortes diárias do trânsito brasileiro. Além disso, em 2001, a média era de 8,5 mortos em motos por dia e que as taxas aumentaram na última década, mais de $800 \%$ de aumento entre 1996 e 2006, de 0,4 para 3,6 por grupos de 100 mil habitantes (GANNE, 2010).

Entre 1998 e 2011, a taxa de óbitos dos motociclistas oscilou de um mínimo de 67,8 mortes por 100 mil motocicletas em 1998, até um máximo de 101,1 por 100 mil motocicletas em 2002, com uma média de 91 óbitos também por 100 mil motocicletas registradas.

Nessa mesma década, o número de vítimas de automóvel oscilou de um mínimo de 30 por 100 mil motocicletas, em 2009, até um máximo de 41,5 por 100 mil motocicletas em 1999. No melhor ano de crescimento dos veículos automotores, 1999, a taxa foi de 10,3\%. Além disso, a mortalidade dos motociclistas foi $146,3 \%$ maior que a dos passageiros de automóveis. Outro dado relevante é que a frota de motocicletas cresceu $491 \%$ no período enquanto as mortes de motociclistas cresceram $610 \%$.

Em outras palavras: $491 \%$ do incremento da mortalidade devem-se ao aumento da frota de motocicletas e o restante (119\%) deve ser atribuído como um aumento do risco motocicleta no trânsito, isto é, maior risco de morte de motociclistas no trânsito (WAISELFISZ JJ, 2013).

Diante disso, o objetivo do presente estudo foi analisar as internações por acidentes com motocicletas no sistema público de saúde da Bahia no período entre 2008 e 2017.

\section{MÉTODOS}

Trata-se de um estudo ecológico transversal, de abordagem descritiva das internações por acidentes com motocicletas na rede pública de saúde do estado da Bahia. Os dados de internações foram obtidos do Sistema de Informações Hospitalares do SUS (SIH/SUS) do Ministério da Saúde.

Estes foram relativos aos internamentos decorrentes do grupo de causas do CID-10 V20-V29 (motociclista traumatizado em acidente de transporte) e selecionados por local de residência e ano de ocorrência, no período entre os anos de 2008 e 2017.

Os dados da população utilizados para cálculo da taxa de mortalidade foram obtidos do último censo demográfico do Instituto Brasileiro de Geografia e Estatística (IBGE, 2010) do ano de 2010. Os coeficientes de incidência foram construídos a partir da razão entre o número de internações hospitalares e a população do censo do IBGE, multiplicado por 100.000 habitantes.

Foi utilizada para tabulação dos dados e cálculos estatísticos a planilha do Programa Microsoft Excel ${ }^{\circledR}$ versão 2010.

O projeto de pesquisa não precisou da aprovação do Comitê de Ética em Pesquisa, pois usou dados secundários de base de dados de domínio público, em concordância com a Resolução do Conselho Nacional de Saúde (CNS) ํㅜ 466/2012.

\section{RESULTADOS}

Entre os anos de 2008 a 2016 foi observado um aumento constante do número total de pacientes internados vítimas de acidentes com motocicletas na Bahia e a cidade de Salvador teve o maior número de pacientes internados e o total de pacientes foi de 45714 ao longo dos dez anos avaliados.

O ano de 2017 apresentou uma redução do número de pacientes internados em relação aos anos de 2016 e 2015. Entre os anos de 2008 e 2017 foi observado um aumento de $322 \%$ de internações registradas (tabela 1). Com base nos dados do IBGE foi possível calcular a frequência de internações por acidentes com motocicletas na Bahia. Em 2008 foi de 13,07 por 100 mil habitantes, atingindo o máximo de 43,61 por 100 mil habitantes em 2016 e terminando a série com 42,15 por 100 mil habitantes. 
Tabela 1 - Total de internações, por município, no Estado da Bahia entre os anos de 2008-2017, (DATASUS).

\begin{tabular}{|c|c|c|c|c|c|c|c|c|c|c|c|}
\hline Internações & 2008 & 2009 & 2010 & 2011 & 2012 & 2013 & 2014 & 2015 & 2016 & 2017 & Total \\
\hline Alagoinhas & 2 & 19 & 62 & 46 & 25 & 9 & 0 & 3 & 14 & 9 & 189 \\
\hline Barreiras & 2 & 1 & 5 & 2 & 14 & 5 & 81 & 69 & 74 & 24 & 277 \\
\hline Brumado & 30 & 34 & 65 & 58 & 54 & 84 & 93 & 123 & 87 & 129 & 757 \\
\hline Camaçari & 0 & 3 & 3 & 0 & 3 & 1 & 1 & 23 & 14 & 7 & 55 \\
\hline $\begin{array}{c}\text { Cruz das } \\
\text { Almas }\end{array}$ & 17 & 46 & 15 & 22 & 23 & 37 & 31 & 14 & 20 & 19 & 244 \\
\hline $\begin{array}{l}\text { Feira de } \\
\text { Santana }\end{array}$ & 254 & 477 & 498 & 724 & 798 & 1001 & 717 & 676 & 852 & 740 & 6737 \\
\hline Guanambi & 1 & 28 & 19 & 27 & 24 & 25 & 57 & 97 & 127 & 39 & 444 \\
\hline Ibotirama & 2 & 5 & 35 & 41 & 70 & 80 & 85 & 140 & 187 & 131 & 776 \\
\hline Ilhéus & 0 & 7 & 11 & 16 & 17 & 9 & 17 & 42 & 10 & 10 & 139 \\
\hline Irecê & 1 & 1 & 0 & 0 & 0 & 0 & 2 & 0 & 0 & 1 & 5 \\
\hline Itaberaba & 55 & 140 & 122 & 164 & 190 & 141 & 248 & 239 & 246 & 254 & 1799 \\
\hline Itabuna & 0 & 2 & 6 & 4 & 7 & 14 & 9 & 0 & 0 & 2 & 44 \\
\hline Itapetinga & 6 & 4 & 12 & 2 & 3 & 18 & 27 & 30 & 42 & 57 & 201 \\
\hline Jacobina & 26 & 41 & 85 & 69 & 56 & 80 & 101 & 128 & 91 & 149 & 826 \\
\hline Jequié & 2 & 11 & 25 & 17 & 14 & 11 & 25 & 23 & 17 & 31 & 176 \\
\hline Juazeiro & 300 & 602 & 802 & 926 & 808 & 846 & 1054 & 1037 & 699 & 416 & 7490 \\
\hline $\begin{array}{c}\text { Paulo } \\
\text { Afonso }\end{array}$ & 0 & 0 & 0 & 0 & 0 & 0 & 0 & 0 & 2 & 0 & 2 \\
\hline $\begin{array}{l}\text { Porto } \\
\text { Seguro }\end{array}$ & 8 & 6 & 19 & 26 & 207 & 269 & 308 & 364 & 365 & 348 & 1920 \\
\hline $\begin{array}{l}\text { Ribeira do } \\
\text { Pombal }\end{array}$ & 0 & 0 & 5 & 10 & 10 & 5 & 1 & 2 & 5 & 5 & 43 \\
\hline Salvador & 975 & 1264 & 1378 & 1578 & 1772 & 1584 & 2055 & 1862 & 1766 & 2058 & 16292 \\
\hline $\begin{array}{l}\text { Santa Maria } \\
\text { da Vitória }\end{array}$ & 2 & 18 & 45 & 34 & 75 & 88 & 126 & 245 & 191 & 221 & 1045 \\
\hline $\begin{array}{l}\text { Santo } \\
\text { Antônio de } \\
\text { Jesus }\end{array}$ & 51 & 39 & 28 & 39 & 31 & 36 & 30 & 29 & 41 & 39 & 363 \\
\hline Seabra & 7 & 12 & 25 & 48 & 75 & 41 & 46 & 89 & 187 & 195 & 725 \\
\hline $\begin{array}{l}\text { Senhor do } \\
\text { Bonfim }\end{array}$ & 10 & 7 & 6 & 10 & 13 & 14 & 24 & 18 & 42 & 13 & 157 \\
\hline Serrinha & 4 & 5 & 18 & 35 & 40 & 121 & 126 & 158 & 262 & 294 & 1063 \\
\hline $\begin{array}{l}\text { Teixeira de } \\
\text { Freitas }\end{array}$ & 3 & 2 & 7 & 8 & 10 & 339 & 494 & 441 & 441 & 418 & 2163 \\
\hline Valença & 0 & 1 & 2 & 1 & 7 & 10 & 4 & 5 & 8 & 2 & 40 \\
\hline $\begin{array}{l}\text { Vitória da } \\
\text { Conquista }\end{array}$ & 72 & 83 & 127 & 159 & 149 & 154 & 187 & 204 & 316 & 291 & 1742 \\
\hline Total & 1830 & 2858 & 3425 & 4066 & 4495 & 5022 & 5949 & 6061 & 6106 & 5902 & 45714 \\
\hline
\end{tabular}

Fonte: Dados da pesquisa, 2018.

O tempo médio de internação dos pacientes internados na Bahia por acidentes com motocicletas foi de 31,12 dias. A taxa de mortalidade variou de 5,23\% em 2008 e atingiu o máximo no ano de 11,94 em 2011. A média da taxa de mortalidade ao longo dos dez anos avaliados foi de 9,54\% (gráfico 1).

Assim, houve aproximadamente, 4361 mortes no período no estado da Bahia no período de 2008 a 2017. A taxa de mortalidade por 100 mil habitantes variou de 1,27 em 2008 a 4,09 em 2017. 
Gráfico 1 - Taxa de mortalidade por ano.

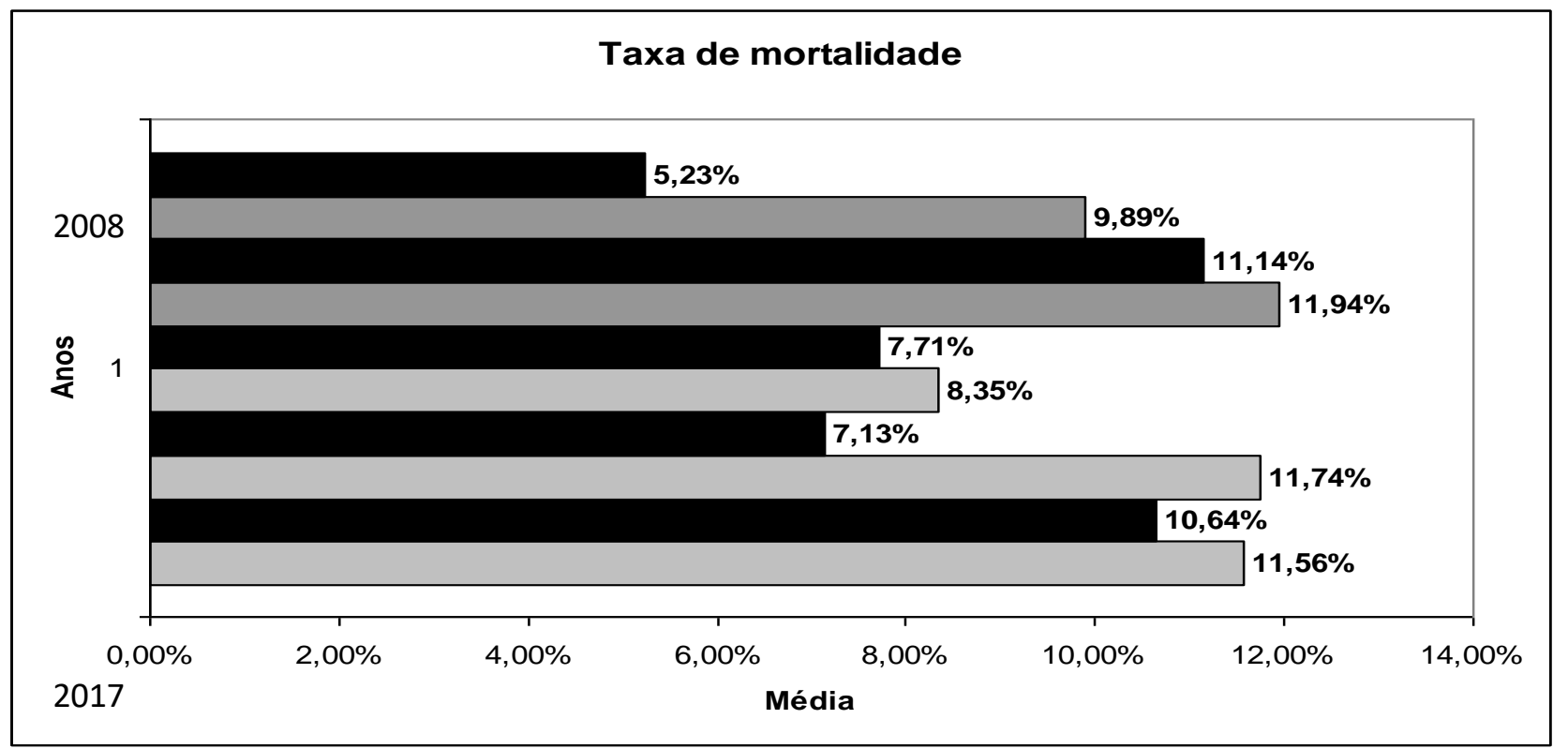

Fonte: Dados da pesquisa, 2018.

A análise dos dados revelou que o custo médio de cada uma das internações foi de 4.545,62 reais e um custo total de aproximadamente 207 milhões e 798 mil reais. A distribuição por faixas etárias mostra que 26756 pacientes (58,53\%) tinham idade entre 20 a 39 anos. $900(1,97 \%)$ crianças entre 0 a 14 anos e 1855 $(4,06 \%)$ idosos foram internadas por acidente de motocicletas no estado da Bahia no período avaliado (tabela 2).

Tabela 2 - Distribuição por faixa etária.

\begin{tabular}{cccccccccccc}
\hline Faixa Etária & $\mathbf{2 0 0 8}$ & $\mathbf{2 0 0 9}$ & $\mathbf{2 0 1 0}$ & $\mathbf{2 0 1 1}$ & $\mathbf{2 0 1 2}$ & $\mathbf{2 0 1 3}$ & $\mathbf{2 0 1 4}$ & $\mathbf{2 0 1 5}$ & $\mathbf{2 0 1 6}$ & $\mathbf{2 0 1 7}$ & TOTAL \\
\hline Menor 1 ano & 2 & 8 & 4 & 5 & 7 & 3 & 4 & 9 & 3 & 5 & 50 \\
\hline 1 a 4 anos & 10 & 16 & 20 & 27 & 22 & 22 & 39 & 40 & 34 & 45 & 275 \\
\hline 5 a 9 anos & 29 & 44 & 48 & 42 & 48 & 44 & 50 & 68 & 50 & 47 & 470 \\
\hline 10 a 14 anos & 52 & 64 & 93 & 77 & 120 & 126 & 147 & 136 & 114 & 124 & 1053 \\
\hline 15 a 19 anos & 231 & 338 & 409 & 522 & 543 & 651 & 714 & 765 & 757 & 766 & 5696 \\
\hline 20 a 29 anos & 782 & 1135 & 1320 & 1424 & 1489 & 1594 & 1929 & 1921 & 1906 & 1819 & 15319 \\
\hline 30 a 39 anos & 405 & 660 & 809 & 1019 & 1197 & 1242 & 1529 & 1522 & 1566 & 1488 & 11437 \\
\hline 40 a 49 anos & 205 & 374 & 402 & 560 & 591 & 719 & 839 & 883 & 938 & 846 & 6357 \\
\hline 50 a 59 anos & 91 & 144 & 192 & 239 & 293 & 337 & 445 & 485 & 488 & 488 & 3202 \\
60 a 69 anos & 36 & 60 & 82 & 107 & 130 & 179 & 192 & 153 & 198 & 201 & 1338 \\
\hline 70 a 79 anos & 14 & 20 & 43 & 51 & 52 & 70 & 57 & 73 & 48 & 70 & 498 \\
\hline 80 anos e mais & 9 & 2 & 0 & 1 & 1 & 1 & 1 & 1 & 1 & 2 & 19 \\
\hline TOTAL & 1866 & 2865 & 3422 & 4074 & 4493 & 4988 & 5946 & 6056 & 6103 & 5901 & 45714 \\
\hline Fon
\end{tabular}

Fonte: Dados da pesquisa, 2018.

Do total de pacientes internados 38908 foram do sexo masculino representando $85,12 \%$ do total e 6806 do sexo feminino representando $14,88 \%$ do total. 


\section{DISCUSSÃO}

Os acidentes de trânsito, especialmente os que envolvem motociclistas, representam grave e emergente problema de saúde pública no Brasil. Outros estudos publicados nos últimos anos corroboram os achados do presente trabalho. Estudo publicado com avaliação de vítimas de acidentes motociclísticos no Paraná revelam que os homens eram $80,6 \%$ das vítimas e 38,7\% tinham entre 20 e 29 anos e $25,8 \%$ apresentaram fratura exposta nos membros inferiores (MENOLLI GA, MARTINS EAP. 2018). Além disso, em estudo para avaliação de pacientes com diagnóstico de traumatismo crânio encefálico no Maranhão, identificou-se que $64,3 \%$ dos pacientes haviam sido vítimas de acidentes de trânsito e $44,6 \%$ do total eram motociclistas (MARTINS A C P, ALMEIDA RVD, ÁVILA ITS, SILVA WB, 2018)

Os resultados apresentados mostram que os traumas envolvendo acidentes de motocicleta representam um sério problema de saúde pública no Estado da Bahia. A distribuição geográfica apresentada na tabela 1 revelam que a capital, Salvador, concentra a maior parte das vítimas.

A taxa de mortalidade por acidente de motocicletas no Brasil aumentou $800 \%$, variando de 0,5 para 4,5/100.000 habitantes entre 1996 e 2009, um incremento médio anual de 19\% (MARTINS et al, 2013).

Em nossa série, no Estado da Bahia, a taxa de mortalidade por 100 mil habitantes variou de 1,27 em 2008 a 4,09 em 2017. Importante observar que esses óbitos se referem apenas aqueles pacientes que morreram nos hospitais, estando excluídos os que sofreram acidentes fatais nas vias públicas e não conseguiram receber atendimento hospitalar. Assim, é possível supor que essas taxas sejam ainda maiores.

Outro dado revelado pelo trabalho foi o longo período médio de internação dos pacientes envolvidos em acidentes com motocicletas no estado da Bahia. Em média a internações duraram cerca de 31 dias. No ano de 2013 o tempo médio de internação no Brasil relacionados a todos os acidentes de transporte terrestre foi de apenas 6,3 dias por paciente (ANDRADE, JORGE, 2017).

No mesmo estudo foi mostrado que do total de vítimas, $78,2 \%$ eram indivíduos do sexo masculino e $48,6 \%$ na faixa etária de 20 a 39 anos. Em nossa série observamos números significativamente maiores nas duas variáveis. $58,53 \%$ das vítimas estavam na faixa etária compreendida entre 20 a 39 anos e $85,12 \%$ eram do sexo masculino. Esses dados revelam que há um maior risco de acidentes para homens e indivíduos jovens em idade produtiva o que permite concluir que os custos envolvidos são bem maiores do que aqueles apurados. Se considerarmos a perda de produtividade, afastamento do trabalho, cuidados de terceiros e outros custos indiretos fica claro que o custo econômico e social deste grupo de doenças é muito maior do que o custo direto. Em 2013, o gasto total das internações por acidentes de transporte terrestre no Brasil alcançou $\mathrm{R} \$ 231.469 .333,13$, com 1.072 .557 dias de permanência e média de 6,3 dias de internação por paciente.

A principal limitação do estudo diz respeito ao fato de que os dados disponíveis não fornecem uma visão dos pacientes atendidos na rede privada de saúde. Segundo dados da Agência Nacional de Saúde Suplementar (ANS) em outubro de 2017, a Bahia tinha 1.577 .743 milhões de beneficiários na Saúde Suplementar (ANS, 2017).

Neste mesmo ano, a Bahia se tornou o primeiro estado do país a tornar os acidentes de trânsito em doenças de notificação obrigatória (DETRAN BA, 2017) tornando os futuros estudos sobre este sério agravo de saúde mais representativos da realidade pela esperada diminuição das subnotificações.

\section{CONCLUSÃO}

O estudo mostra a gravidade dos acidentes de motocicletas atendidos no Sistema Único de Saúde na Bahia comprometendo principalmente homens jovens, em idade produtiva e com alto custo e taxa de mortalidade. Esses dados reforçam a necessidade de medidas institucionais para redução do número de acidentes e novos estudos são importantes para determinar as sequelas que eventualmente acometem os sobreviventes. 


\section{REFERÊNCIAS}

1. Agência Nacional de Saúde Suplementar (ANS), Sala de Situação, 2017.

2. ANDRADE SSCA, JORGE MHPM. Internações hospitalares por lesões decorrentes de acidente de transporte terrestre, Epidemiol. Serv. Saude, Brasília, 26(1):31-38, jan-mar 2017.

3. BACCHIERI G, BARROS AJD, Acidentes de trânsito no Brasil, Rev Saúde Pública 2011;45(5):949-63

4. GANNE N. Estudo sobre acidentes de trânsito envolvendo motocicletas, Rev Pan-Amaz Saude 2010; 1(3):19-24.

5. Departamento Estadual de Trânsito da Bahia (DETRAN BA), Acidente de trânsito vira doença de notificação obrigatória na Bahia, 2017.

6. LEI № 9.503, DE 23 DE SETEMBRO DE 1997. Código de Trânsito Brasileiro. Presidência da República

7. The Global status report on road safety 2018. World Health Organization. 2018.

8. MARTINS ACP, Almeida RVD, ÁVILA ITS. Perfil clínico e epidemiológico de pacientes acometidos por trauma cranioencefálico assistidos em hospital público de grande porte. REAS, Revista Eletrônica Acervo Saúde, 2018. Vol. 10 (1), 2065-2072.

9. MARTINS ET et al, Mortalidade por acidentes de motocicleta, Rev Saúde Pública 2013;47(5):931-4.

10. MARTINEZ A. Motocicletas: o conflito entre a agilidade e segurança. Rev ABRAMET. 2006;(48):29-31

11. MENOLLI GA, MARTINS EAP. Caracterização do atendimento pré-hospitalar a vítimas de acidente motociclísticos encaminhadas para um hospital de grande porte do norte do Paraná. REAS, Revista Eletrônica Acervo Saúde, 2018. Vol. 10 (6), 2280-2287.

12. ANDRADE SSCA, JORGE MHPM. Internações hospitalares por lesões decorrentes de acidente de transporte terrestre no Brasil, 2013: permanência e gastos. Epidemiol. Serv. Saude, Brasília, 26(1):31-38, jan-mar 2017.

13.WAISELFISZ JJ. MAPA DA VIOLÊNCIA 2013: Acidentes de Trânsito e Motocicletas. 2013. 